PESQUIMAT 22(2): 85-105 (2019)

http://dx.doi.org/10.15381/pesquimat.v22i2.17236
ISSN:1560-912X/ ISSN-E:1609-8439

Facultad de Ciencias Matemáticas - UNMSM

\title{
Acerca del Teorema de Malgrange
}

\author{
Percy Braulio Fernández Sánchez ${ }^{1}$ Danny Joel Apaza Nuñez ${ }^{2}$
}

Resumen: En este artículo daremos una demostración del Teorema de Malgrange sobre un subcuerpo $\mathbb{K}$ de los complejos, esta demostración es debido a Moussu. Este resultado es importante en la teoria de foliaciones y demuestra que todas las foliaciones holomorfas de codimensión uno con un conjunto singular pequeño tiene integral primera holomorfa.

Palabras clave: 1-formas; divisores de cero; explosión.

\section{About of Malgrange Theorem}

\begin{abstract}
In this article we will prove of Malgrange Theorem on a subfield $\mathrm{K}$ of the complex numbers, this demonstration is due to Moussu. This result is important in the theory of foliations and shows that all holomorphic foliations of Codimension one with a small singular set has holomorphic first integral.
\end{abstract}

Keywords: 1-forms; zero divisors; blowing up.

Recibido: 20/10/2019. Aceptado: 18/11/2019. Publicado online: 26/12/2019.

(CLos autores. Este artículo es publicado por la Revista PESQUIMAT de la Facultad de Ciencias Matemáticas, Universidad Nacional Mayor de San Marcos. Este es un artículo de acceso abierto, distribuido bajo los términos de la licencia Creative Commons Atribucion-No Comercia-Compartir Igual 4.0 Internacional.(http://creativecommons.org/licenses/by-nc-sa/4.0/) que permite el uso no comercial, distribución y reproducción en cualquier medio, siempre que la obra original sea debidamente citada. Para información, por favor póngase en contacto con revistapesquimat.matematica@unmsm.edu.pe

\footnotetext{
${ }^{1}$ PUCP, Facultad de Ciencias e Ingeniería. e-mail: pefernan@pucp.edu.pe

${ }^{2}$ UNALM, Facultad de Ciencias. e-mail: dannyapaza@lamolina.edu.pe
} 


\section{Introducción}

Este trabajo es desarrollado a partir de la tesis de maestría: El teorema de De Rham- Saito y el Teorema de Frobenius Singular [1], donde mostramos en detalle la demostración del

\section{Teorema de Malgrange sobre un subcuerpo $\mathbb{K}$ de los complejos}

Sea $\omega$ un germen en $0 \in \mathbb{C}^{n}$ una 1-forma diferencial holomorfa de codimensión uno con coeficientes en $\mathbb{K}\{x\}$, que verifica:

i) $\omega \wedge d \omega=0$

ii) $\operatorname{Codim}(\operatorname{Sing} \omega) \geq 3$.

Entonces $\omega$ es $\mathbb{K}$-integrable si existen dos gérmenes $f, g \in \mathbb{K}\{x\}$ tales que $\omega=g d f$ con $g(0) \neq 0$.

Nuestro principal objetivo es realizar la demostración del Teorema de Malgrange sobre un subcuerpo de los números complejos [6].

\section{Preliminares}

En esta sección presentaremos algunos conceptos previos sobre el anillo de series de potencias, dominio, anillo local, ideal maximal los cuales no demostraremos, el lector interesado puede encontrar las pruebas en [2]. Por otro lado, demostramos el Lema de Poincaré relativamente graduado, además usaremos el Lema de Poincaré graduado clásico [4], también enunciaremos el teorema de funciones compuestas [7] y utilizamos los conceptos de singularidades, transversabilidad y explosiones.

\section{Anillo de Series de Potencias}

Sea $\mathbb{K}$ un cuerpo, $X_{1}, \ldots, X_{r}$ indeterminadas sobre $\mathbb{K}$ y denotemos por

$$
\mathcal{R}=\mathbb{K}\left[\left[X_{1}, \ldots, X_{r}\right]\right]
$$

al conjunto de todas las sumas formales de tipo

$$
f=\sum_{i=0}^{\infty} P_{i}=P_{0}+P_{1}+P_{2}+\cdots
$$

donde cada $P_{i}$ es un polinomio homogéneo de grado $i$ en las variables $X_{1}, \ldots, X_{r}$ con coeficientes en $\mathbb{K}$, consideraremos al polinomio cero como un polinomio homogéneo de cualquier grado.

Sean $f=P_{0}+P_{1}+\cdots, g=Q_{0}+Q_{1}+\cdots$ elementos de $\mathcal{R}$.

Por definición $f=g \Leftrightarrow P_{i}=Q_{i}$ para todo $i \in \mathbb{N}$.

En $\mathcal{R}$ definimos las siguientes operaciones:

$$
\begin{aligned}
f+g & =\sum_{i=0}^{\infty}\left(P_{i}+Q_{i}\right) \\
f . g & =\sum_{i=0}^{\infty} \sum_{j=0}^{i} P_{i} Q_{i-j}
\end{aligned}
$$

Con estas operaciones, $\mathcal{R}$ es un anillo con unidad, llamado el anillo de series de potencias formales en $r$ variables con coeficientes en $\mathbb{K}$. 
El anillo $\mathcal{R}$ tiene como subanillos al campo $\mathbb{K}$ y al anillo de polinomios $\mathbb{K}\left[X_{1}, \ldots, X_{r}\right]$. Los elementos de $\mathcal{R}$ tienen la forma:

$$
f=\sum_{i=0}^{\infty} \sum_{i_{1}+\cdots+i_{r}=i} a_{i_{1}, \ldots, i_{r}} X_{1}^{i_{1}} \cdots X_{r}^{i_{r}} ; \quad a_{i_{1}, \ldots, i_{r}} \in \mathbb{K} .
$$

Si $\mathbb{K}$ es el cuerpo de números reales ó complejos, podemos asumir al subanillo $A=\mathbb{K}\left\{X_{1}, \ldots, X_{r}\right\}$ de $\mathcal{R}$ que consta de las series de potencias absolutamente convergentes en una vecindad del origen $(0, \ldots, 0) \in \mathbb{K}^{n}$. En otras palabras, los elementos de $A$ son las series $\sum_{i=0}^{\infty} \sum_{i_{1}+\cdots+i_{r}=i} a_{i_{1}, \ldots, i_{r}} X_{1}^{i_{1}} \cdots X_{r}^{i_{r}}$ para las cuales existe un número real positivo $\rho$ (dependiendo de $f$ ) tal que las series

$$
\sum_{i=0}^{\infty} \sum_{i_{1}+\cdots+i_{r}=i}\left|a_{i_{1}, \ldots, i_{r}}\right| \rho^{i_{1}+\cdots+i_{r}}
$$

son convergentes. Además

- El elemento $f=\sum_{i=0}^{\infty} P_{i}$ en $\mathcal{R}$ es invertible si, y solamente si, $P_{0} \neq 0$.

- Dos elementos $f$ y $g$ en $\mathcal{R}$ son asociados si existe una unidad, esto es, un elemento invertible $u$ tal que $f=u \cdot g$.

Definición 2.1 Sea $f \in \mathcal{R} \backslash\{0\}$. Suponga que

$$
f=P_{n}+P_{n+1}+\cdots
$$

donde cada $P_{j}$ es un polinomio homogéneo de grado $j$ y $P_{n} \neq 0$. el polinomio homogéneo $P_{n}$ es llamado la forma inicial de $f$. El entero $n$ es llamado la multiplicidad de $f$ y es denotado por $\operatorname{mult}(f)$. Si $f=0$, tendremos que $\operatorname{mult}(f)=\infty$.

- $f \in \mathcal{R}$ es invertible si y solamente si $\operatorname{mult}(f)=0$.

- Sea $f, g \in \mathcal{R}$, la multiplicidad de series de potencias tiene las siguientes propiedades:

i) $\operatorname{mult}(f \cdot g)=\operatorname{mult}(f)+\operatorname{mult}(g)$,

ii) $\operatorname{mult}(f \pm g) \geq \operatorname{mín}\{\operatorname{mult}(f)$, $\operatorname{mult}(g)\}$, la igualdad se da cuando $\operatorname{mult}(f) \neq \operatorname{mult}(g)$.

- El anillo $\mathcal{R}$ es un dominio.

Denotemos por $\mathcal{R}$ al anillo $\mathbb{K}\left[\left[X_{1}, \ldots, X_{r}\right]\right]$ y por $\mathcal{M}_{\mathcal{R}}$ su ideal maximal, $\mathcal{M}_{\mathcal{R}}^{i}$ la $i$-ésima potencia del ideal $\mathcal{M}_{\mathcal{R}}$, y ponemos $\mathcal{M}_{\mathcal{R}}^{0}=\mathcal{R}$, Además se tiene:

- $\mathcal{R}$ es un anillo local.

- El ideal $\mathcal{M}_{\mathcal{R}}$ es el único ideal maximal de $\mathcal{R}$ y es tal que

$$
\bigcap_{i \in \mathbb{N}} \mathcal{M}_{\mathcal{R}}^{i}=\{0\}
$$


- Sea $\rho>1$ un número real y considere la siguiente aplicación

$$
\begin{aligned}
d: \mathcal{R} \times \mathcal{R} & \rightarrow \mathbb{R} . \\
(f, g) & \mapsto d(f, g)=\rho^{-\operatorname{mult}(f-g)}
\end{aligned}
$$

El par $(\mathcal{R}, d)$ es un espacio métrico completo.

- Si $(\mathcal{R}, d)$ es un espacio métrico completo entonces $(\underbrace{\mathcal{R} \times \cdots \times \mathcal{R}}_{n \text {-veces }}, d^{\prime})$ es un espacio métrico completo, donde $d^{\prime}=\sum d_{i}, \quad i=1, \ldots, n$.

Corolario 2.1 $M \in \mathbb{K}[[X]]^{p \times p}, M=M_{0}+M_{1}+\cdots$. Si $\operatorname{det} M_{0} \neq 0$; existe $N \in \mathbb{K}[[X]]^{p \times p}$ tal que $N M=I$ donde $I$ es la matriz identidad.

Demostración. Ver [1].

\section{El Teorema de Frobenius Formal sobre $\mathbb{K}$}

Proposición 3.1 La sucesión $X_{k}=M_{k} \ldots M_{1}$ con $M_{k}=I+U_{k}$ donde $I$ es la matriz identidad, $U_{k}=\left(u_{i, j}^{k}\right)$ es una matriz de $\mathbb{K}[[X]]^{p \times p}$ y $u_{i, j}^{k}$ son polinomios homogéneos de grado $k$, con la métrica definida anteriormente, es convergente.

Demostración. La prueba que realizaremos es similar a la demostración de la proposición 4.2.5 ([1], p. 59), para verificar la completitud usaremos una definición equivalente a la de sucesión de Cauchy, es decir, para todo $\epsilon>0$, existe $k \in \mathbb{N}$ tal que $n>k$ entonces $\left|X_{k+n}-X_{k}\right|<\epsilon$, donde $X_{k}=M_{k} \ldots M_{1}$. Para ello necesitamos verificar que

$$
\operatorname{mult}\left(\left(M_{k+n} \ldots M_{k+1} M_{k} M_{k-1} \ldots M_{1}\right)-\left(M_{k} M_{k-1} \ldots M_{1}\right)\right) \geq k+1, k \geq 1, n \geq 1,
$$

esto quiere decir que todos los términos de la matriz $X_{n+k}-X_{k}$ tienen multiplicidad mayor o igual que $k+1$.

1. Caso $k=1$ y $n \geq 1$

$$
\operatorname{mult}\left(M_{n+1} \ldots M_{1}-M_{1}\right) \geq 2
$$

de esto se obtiene lo que queríamos.

$$
\begin{aligned}
M_{n+1} \ldots M_{2} & =\left(I+U_{n+1}\right) \ldots\left(I+U_{2}\right) \\
& =I+\sum_{j=1}^{n} \sum_{i_{1}<\cdots<i_{j}} U_{i_{j}} U_{i_{j-1}} \ldots U_{i_{1}} \\
M_{n+1} \ldots M_{2} M_{1} & =M_{1}+\left(\sum_{j=1}^{n} \sum_{\substack{i_{1}<\cdots<i_{j} \\
\left\{i_{1}, \ldots, i_{j}\right\} \subset\{2, \ldots, n+1\}}} U_{i_{j}} U_{i_{j-1}} \ldots U_{i_{1}}\right) M_{1}
\end{aligned}
$$

restamos $M_{1}$

$$
\left(M_{n+1} \ldots M_{2} M_{1}\right)-M_{1}=\left(\sum_{j=1}^{n} \sum_{\substack{i_{1}<\cdots<i_{j} \\\left\{i_{1}, \ldots, i_{j}\right\} \subset\{2, \ldots, n+1\}}} U_{i_{j}} U_{i_{j-1}} \ldots U_{i_{1}}\right) M_{1}
$$

luego

$$
\operatorname{mult}\left(\left(M_{n+1} \ldots M_{2} M_{1}\right)-M_{1}\right) \geq 2 .
$$


2. Caso general

$$
M_{k+n} \ldots M_{k+1}=I+\sum_{j=1}^{k+n-1} \sum_{\substack{i_{1}<\ldots<i_{j} \\\left\{i_{1}, \ldots, i_{j}\right\} \subset\{k+1, \ldots, k+n\}}} U_{i_{j}} U_{i_{j-1}} \ldots U_{i_{1}}
$$

multiplicando por $M_{k} \ldots M_{1}$ y restando $M_{k} \ldots M_{1}$

$$
\left(M_{k+n} \ldots M_{k+1} M_{k} \ldots M_{1}\right)-\left(M_{k} \ldots M_{1}\right)=\left(\sum_{j=1}^{k+n-1} \sum_{\substack{i_{1}<\ldots<i_{j} \\\left\{i_{1}, \ldots, i_{j}\right\} \subset\{k+1, \ldots, k+n\}}} U_{i_{j}} U_{i_{j-1}} \ldots U_{i_{1}}\right) M_{k} \ldots M_{1}
$$

tomando multiplicidad obtenemos el resultado

$\operatorname{mult}\left(\left(M_{k+n} \ldots M_{k+1} M_{k} \ldots M_{1}\right)-\left(M_{k} \ldots M_{1}\right)\right) \geq k+1$.

Si las entradas de $X_{k}$ son $x_{i j}^{k}$, esto es $X_{k}=\left(x_{i j}^{k}\right)$ tenemos

$$
\operatorname{mult}\left(x_{i j}^{k+n}-x_{i j}^{k}\right) \geq k+1 \quad \forall i, j
$$

luego

$$
d\left(x_{i j}^{k+n}, x_{i j}^{k}\right)=\rho^{-\operatorname{mult}\left(x_{i j}^{k+n}-x_{i j}^{k}\right)} \leq \rho^{-(k+1)}, \quad \rho>1
$$

entonces

$$
\left|X_{k+n}-X_{k}\right|=\sum d\left(x_{i j}^{k+n}, x_{i j}^{k}\right) \leq p^{2} \rho^{-(k+1)}=\epsilon
$$

Por lo tanto la sucesión $\left(X_{k}\right)$ es de Cauchy.

Ahora probaremos un lema que nos servirá en la demostración del Teorema de Frobenius Formal el cual nos ayudará a demostrar el teorema de Frobenius Clásico sobre $\mathbb{K}$.

Para la demostración del siguiente lema usaremos el teorema de De Rham - Saito demostrado en $[2$, p. 45$]$.

Lema 3.1 (Lema de Poincaré relativamente graduado) Sea $\alpha^{k} \in \Omega_{1}^{k}(\mathbb{K})$ tal que $d x_{1} \wedge d x_{2} \wedge \ldots \wedge d x_{p} \wedge d \alpha^{k}=0$, entonces existen polinomios homogéneos de grado $k, u_{1}^{k}, u_{2}^{k}, \ldots, u_{p}^{k} \in$ $\Omega_{0}^{k}$ y $u^{k+1} \in \Omega_{0}^{k+1}$ tales que $\alpha^{k}=d u^{k+1}+u_{1}^{k} d x_{1}+\cdots+u_{p}^{k} d x_{p}$.

Demostración. Haremos la demostración por inducción en el par $(p, k)$ considerando el orden lexicográfico ${ }^{1}$.

Para $k=0, \alpha^{0}$ es cerrada, es decir, si existe $u^{1} \in \Omega_{0}^{1}$ tal que $\alpha^{0}=a_{1} d x_{1}=d\left(a_{1} x_{1}\right)=d u^{1}$.

Suponemos que el lema es verdadero para los pares $\left(p^{\prime}, k^{\prime}\right)<(p, k)$ y $d x_{1} \wedge d x_{2} \wedge \ldots \wedge d x_{p} \wedge d \alpha^{k}=0$. Usando el teorema de De Rham-Saito [2], existen $\beta_{i}^{k-1} \in \Omega_{1}^{k-1}(\mathbb{K})$ tales que:

$$
d \alpha^{k}=\beta_{1}^{k-1} \wedge d x_{1}+\beta_{2}^{k-1} \wedge d x_{2}+\cdots+\beta_{p}^{k-1} \wedge d x_{p}
$$

\footnotetext{
${ }^{1} \mathrm{El}$ orden lexicográfico es usado para ordenar el modo en que las palabras aparecen en el diccionario
} 
tomando la derivada exterior

$$
d\left(d \alpha^{k}\right)=d \beta_{1}^{k-1} \wedge d x_{1}+(-1)^{1} \beta_{1}^{k-1} \wedge d\left(d x_{1}\right)+\cdots+d \beta_{p}^{k-1} \wedge d x_{p}+(-1)^{1} \beta_{p}^{k-1} \wedge d\left(d x_{p}\right)
$$

haciendo producto exterior con $d x_{2} \wedge \cdots \wedge d x_{p}$

$$
0=d x_{1} \wedge d x_{2} \wedge \cdots \wedge d x_{p} \wedge d \beta_{1}^{k-1} .
$$

La hipótesis inductiva del lema aplicada a $\beta_{1}^{k-1}$ muestra que existen polinomios $v_{j}^{k-1} \in \Omega_{0}^{k-1}$ y $v^{k} \in \Omega_{0}^{k}$ tales que $\beta_{1}^{k-1}=d v^{k}+v_{1}^{k-1} d x_{1}+\cdots+v_{p}^{k-1} d x_{p}$ reemplazando esta igualdad en la ecuación (1), obtenemos:

$$
\begin{aligned}
d \alpha^{k} & =\left(d v^{k}+v_{1}^{k-1} d x_{1}\right) \wedge d x_{1}, \text { si } p=1 \mathrm{y} \\
d \alpha^{k} & =\left(d v^{k}+\sum_{j=1}^{p} v_{j}^{k-1} d x_{j}\right) \wedge d x_{1}+\sum_{j=2}^{p} \beta_{j}^{k-1} \wedge d x_{j}, \text { si } p>1 .
\end{aligned}
$$

Si $p=1$,

$$
\begin{aligned}
d \alpha^{k} & =\left(d v^{k}+v_{1}^{k-1} d x_{1}\right) \wedge d x_{1} \\
d \alpha^{k} & =d v^{k} \wedge d x_{1} \\
d \alpha^{k}-d v^{k} \wedge d x_{1} & =0 \\
d\left(\alpha^{k}-d v^{k} d x_{1}\right) & =0
\end{aligned}
$$

la igualdad muestra que $\alpha^{k}-v^{k} d x_{1}$ es cerrada. Según el lema de Poincaré graduado clásico, existe $u_{0}^{k+1} \in \Omega_{0}^{k+1}(\mathbb{K})$ tal que

$$
\begin{aligned}
\alpha^{k}-v^{k} d x_{1} & =d u_{0}^{k+1} \\
\alpha^{k} & =d u_{0}^{k+1}+v^{k} d x_{1} .
\end{aligned}
$$

Si $p>1$ escribiremos:

$$
d\left(\alpha^{k}-v^{k} d x_{1}\right)=\left(\sum_{j=2}^{p} v_{j}^{k-1} d x_{j}\right) \wedge d x_{1}+\sum_{j=2}^{p} \beta_{j}^{k-1} \wedge d x_{j} .
$$

Así, haciendo el producto exterior con $d x_{2} \wedge \cdots \wedge d x_{p}$ obtenemos

$$
d\left(\alpha^{k}-v^{k} d x_{1}\right) \wedge d x_{2} \wedge \cdots \wedge d x_{p}=0 .
$$

Utilizamos la hipótesis $p^{\prime}<p$, es decir, existen $v_{j}^{k} \in \Omega_{0}^{k}(\mathbb{K})$ y $v^{k+1} \in \Omega_{0}^{k+1}(\mathbb{K})$

$$
\begin{aligned}
\alpha^{k}-v^{k} d x_{1} & =d v^{k+1}+v_{2}^{k} d x_{2}+\cdots+v_{p}^{k} d x_{p} \\
\alpha^{k} & =d v^{k+1}+v^{k} d x_{1}+v_{2}^{k} d x_{2}+\cdots+v_{p}^{k} d x_{p}
\end{aligned}
$$

Teorema 3.2 (Teorema de Frobenius Formal sobre $\mathbb{K}$ (No singular)) Sea $\Theta=\left\{\omega_{1}, \omega_{2}, \ldots, \omega_{p}\right\} \subset \hat{\Omega}_{1}(\mathbb{K})$ verificando las condiciones:
i) $\omega_{1} \wedge \omega_{2} \wedge \cdots \wedge \omega_{p} \wedge d \omega_{i}=0$ para $i=1, \ldots, p$. (Integrabilidad)
ii) $\omega_{1}(0) \wedge \omega_{2}(0) \wedge \cdots \wedge \omega_{p}(0) \neq 0$.
(Regularidad) 
Entonces $\Theta$ es formalmente integrable sobre $\mathbb{K}$, esto es, existen $\hat{f}_{i}, \hat{g}_{i, j} \in \mathbb{K}[[x]]$ para $i, j=1, \ldots, p$ tales que $\hat{g}_{i, j}(0)=\delta_{i, j}$,

$$
\omega_{i}=\hat{g}_{i, 1} d \hat{f}_{1}+g_{i, 2} d \hat{f}_{2}+\cdots+g_{i, p} d \hat{f}_{p}, \text { para } i=1, \ldots, p .
$$

Demostración. Por un cambio $\mathbb{K}$-lineal de coordenadas, podemos asumir $\omega_{i}(0)=\omega_{i}^{0}=d x_{i}$ para $i=1, \ldots, p$.

Construiremos los $\hat{a}_{i, j} \in \mathbb{K}[[x]], i, j=1, \ldots, p$, tales que $\hat{a}_{i, j}(0)=\delta_{i, j}$ y las 1 -formas $\bar{\omega}_{i}=\hat{a}_{i, 1} \omega_{1}+\cdots+\hat{a}_{i, p} \omega_{p}$ son cerradas; es decir: $\bar{\omega}_{i}=d \hat{f}_{i}, \hat{f}_{i} \in \mathbb{K}[[x]]$. La matriz $M=\left(\hat{a}_{i, j}\right)_{i, j=1, \ldots, p}$ es un elemento de $G L(p, \mathbb{K}[[x]])$ de inversa $\left(\hat{g}_{i, j}\right)_{i, j=1, \ldots, p}$. Por construcción de $\operatorname{los} \hat{a}_{i, j} \in \mathbb{K}[[x]], \hat{g}_{i, j}(0)=\delta_{i, j} \mathrm{y}$

$$
\omega_{i}=\hat{g}_{i, 1} d \hat{f}_{1}+\cdots+\hat{g}_{i, p} d \hat{f}_{p}
$$

Suponiendo que existe $k>0$ tal que para $i=1, \ldots, p$, la forma $\omega_{i}=\omega_{i}^{0}+\omega_{i}^{1}+\cdots+\omega_{i}^{s}+\cdots$ verifica $d \omega_{i}^{s}=0$ para $s<k$. Esta condición se cumple para $k=1$. De la $i$-ésima condición de integrabilidad

$$
\begin{aligned}
\omega_{1} & \wedge \omega_{2} \wedge \ldots \wedge \omega_{p} \wedge d \omega_{i}=0 \\
\left(\omega_{1}^{0}+\omega_{1}^{1}+\cdots\right) \wedge \cdots \wedge\left(\omega_{p}^{0}+\omega_{p}^{1}+\cdots\right) \wedge\left(d \omega_{i}^{k}+\cdots\right) & =0
\end{aligned}
$$

nos quedamos con el primer producto no nulo

$$
d x_{1} \wedge d x_{2} \wedge \cdots \wedge d x_{p} \wedge d \omega_{i}^{k}=0
$$

Según el lema 3.1 , existen los $u_{i, j}^{k} \in \mathbb{K}[[x]]$ y $u^{k+1} \in \mathbb{K}[[x]]$ tales que:

$$
\begin{aligned}
\omega_{i}^{k} & =d u^{k+1}+u_{i, 1}^{k} d x_{1}+\cdots+u_{i, p}^{k} d x_{p} \\
\omega_{i}^{k}-u_{i, 1}^{k} d x_{1}-\cdots-u_{i, p}^{k} d x_{p} & =d u^{k+1} \\
\omega_{i}^{k}-u_{i, 1}^{k} d x_{1}-\cdots-u_{i, p}^{k} d x_{p} & =\bar{\omega}_{i}^{k}
\end{aligned}
$$

la 1 -forma $\bar{\omega}_{i}^{k}=\omega_{i}^{k}+u_{i, 1}^{k} d x_{1}+\cdots+u_{i, p}^{k} d x_{p}$ es exacta para $i=1, \ldots, p$. Puesto que los $u_{i, j}^{k}$ son homogéneos de grado $k$, de la proposición 3.1 la matriz $M=\lim _{k \rightarrow \infty}\left(M_{k} M_{k-1} \ldots M_{1}\right)$, con $M_{k}=I+\left(u_{i, j}^{k}\right)$ está bien definida con $M(0)=I$. Es un elemento de $G L(p, \mathbb{K}[[x]])$ de inversa $\left(\hat{g}_{i, j}\right)_{i, j=1, \ldots, p}$. Por construcción, los $\hat{a}_{i, j}$ verifican las propiedades requeridas.

Para el siguiente teorema necesitamos fijar algunas notaciones.

Sea $X$ un germen de espacio analítico irreducible de dimensión pura $n ; \mathcal{O}_{X}$ denota al anillo de germenes de funciones holomorfas en $X$, si $X=\mathbb{C}^{n}$, escribiremos $\mathcal{O}_{n}$ en lugar de $\mathcal{O}_{X}$, $f:\left(f_{1}, \ldots, f_{p}\right): X \rightarrow\left(\mathbb{C}^{p}, 0\right)$ un germen de aplicación analítica y definimos por Sing $(f)$ al conjunto de puntos donde $f$ no es una sumersión. Observe que Sing $(f)$ es un germen de variedad analítica en $X$.

Denotemos por $\operatorname{prof}(\operatorname{Sin} g(f))$ la profundidad del ideal $I_{f}=(f, d f)$ de $\mathcal{O}_{X}$ asociado a $\operatorname{Sing}(f)$

Ahora enunciaremos el teorema de funciones compuestas que nos servirá para terminar la prueba del teorema de Frobenius clásico sobre $\mathbb{K}$ omitiremos la prueba, sin embargo, para una prueba detallada ver [teorema $2,[7]$, p. 1237]. 
Teorema 3.3 Con las consideraciones anteriores sea $\varphi$ un elemento de $\mathcal{O}_{X}$. Si $\operatorname{prof}(\operatorname{Sing}(f)) \geq 2$, las siguientes condiciones son equivalentes:

1. $d \varphi \wedge d f_{1} \wedge \ldots \wedge d f_{p}=0$ en $X \backslash \operatorname{Sing}(f)$;

2. Existe $\psi \in \mathcal{O}_{p}$ tal que $\varphi=\psi \circ f$;

3. Existe un germen de aplicación $\psi$ en $0 \in \mathbb{C}^{p}$ tal que $\varphi=\psi_{\circ} f$.

\section{El Teorema de Frobenius Clásico sobre $\mathbb{K}$}

Sean $\omega_{1}, \omega_{2}, \ldots, \omega_{p} \in \Omega_{1}(\mathbb{K})$ que verifican las condiciones:

- $\omega_{1} \wedge \omega_{2} \wedge \ldots \wedge \omega_{p} \wedge d \omega_{i}=0$ para $i=1,2, \ldots, p$

- $\omega_{1}(0) \wedge \omega_{2}(0) \wedge \ldots \wedge \omega_{p}(0) \neq 0$.

Como vimos anteriormente podemos elegir las coordenadas $\left(x_{1}, x_{2}, \ldots, x_{n}\right)$ del punto $x \in \mathbb{C}^{n}$ tales que $\omega_{i}(0)=d x_{i}$ para $i=1,2, \ldots, p$ y escribimos:

$$
x^{\prime}=\left(x_{1}, x_{2}, \ldots, x_{p}\right), x^{\prime \prime}=\left(x_{p+1}, x_{p+2}, \ldots, x_{n}\right), x=\left(x^{\prime}, x^{\prime \prime}\right)
$$

Según el teorema clásico de Frobenius [Teorema 4.1.4, [1], p. 55], el sistema de Pfaff

$$
\omega_{1}=\omega_{2}=\cdots=\omega_{p}=0
$$

define una foliación holomorfa $\mathcal{F}$ de codimensión $p$, en un polidisco $\Delta$ centrado en $0 \in \mathbb{C}^{n}$, que es transverso a los espacios afines $x^{\prime \prime}=$ cte. La hoja $L_{x} \in \mathcal{F}$ pasa por el punto $\left(x^{\prime}, x^{\prime \prime}\right) \in \triangle$ corta $x^{\prime \prime}=0$ en un único punto $\left(\tilde{x}^{\prime}, 0\right)$. Las funciones $h_{i}: \Delta \rightarrow \mathbb{C}$ definidas, para $i=1, \ldots, p$, por:

$$
h_{i}(x)=h_{i}\left(x^{\prime}, x^{\prime \prime}\right)=x_{i}
$$

son integrales primeras holomorfas de $\mathcal{F}$ en $\Delta$. Notemos todavía que $h_{i} \in \mathbb{C}\{x\}$ son gérmenes en 0 . Evidentemente, para $i=1, \ldots, p$ :

$$
h_{i}\left(x^{\prime}, 0\right)=x_{i}, \omega_{1} \wedge \omega_{2} \wedge \ldots \wedge \omega_{p} \wedge d h_{i}=0 .
$$

Diremos que los $h_{i}$ son las integrales primeras naturales del sistema de Pfaff en las coordenadas $\left(x_{1}, x_{2}, \ldots, x_{n}\right)$. Reformulamos el teorema principal como sigue:

Teorema 4.1 (de Frobenius clásico sobre $\mathbb{K}$ ) Sean $\omega_{1}, \omega_{2}, \ldots, \omega_{p} \in \Omega_{1}(\mathbb{K})$ que verifican las condiciones:

- $\omega_{1} \wedge \omega_{2} \wedge \ldots \wedge \omega_{p} \wedge d \omega_{i}=0$ para $i=1,2, \ldots, p$

- $\omega_{i}(0)=d x_{i}$ para $i=1,2, \ldots, p$

Entonces las integrales primeras naturales $h_{i}$ pertenecen a $\mathbb{K}\{x\}$.

Demostración. Según el teorema 3.2 , existen $\hat{f}_{i} \in \mathbb{K}[[x]]$ para $i=1,2, \ldots, p$ tales que:

$$
\omega_{i}(0)=d \hat{f}_{i}(0)=d x_{i}
$$

$\omega_{1}=\sum_{j=1}^{p} \hat{g}_{1, j} d f_{j}, \omega_{2}=\sum_{j=1}^{p} \hat{g}_{2, j} d f_{j}, \ldots, \omega_{p}=\sum_{j=1}^{p} \hat{g}_{p, j} d f_{j}$ como el producto exterior $\omega_{1} \wedge \omega_{2} \wedge \ldots \wedge \omega_{p}=\operatorname{Det}\left(\hat{g}_{i, j}\right)_{i, j=1, \ldots, p} d f_{1} \wedge \ldots \wedge d f_{p}$ tenemos $\omega_{1} \wedge \omega_{2} \wedge \ldots \wedge \omega_{p} \wedge d f_{i}=0$. 
El elemento $\hat{\psi}\left(x^{\prime}\right)=\left(\hat{\psi}_{1}\left(x^{\prime}\right), \hat{\psi}_{2}\left(x^{\prime}\right), \ldots, \hat{\psi}_{p}\left(x^{\prime}\right)\right)$ definida por $\hat{\psi}_{i}\left(x^{\prime}\right)=\hat{f}_{i}\left(x^{\prime}, 0\right)$ que pertenece a $\mathbb{K}\left[\left[x^{\prime}\right]\right]^{p \times p}$ y él verifica $D_{x^{\prime}} \hat{\psi}(0)=I$, donde $I$ es la matriz identidad, en efecto,

$$
\hat{\psi}\left(x^{\prime}\right)=\left(\hat{\psi}_{1}\left(x^{\prime}\right), \hat{\psi}_{2}\left(x^{\prime}\right), \ldots, \hat{\psi}_{p}\left(x^{\prime}\right)\right)=\left(\hat{f}_{1}\left(x^{\prime}, 0\right), \ldots, \hat{f}_{p}\left(x^{\prime}, 0\right)\right)
$$

haciendo $\varphi\left(x^{\prime}\right)=\left(x^{\prime}, 0\right)$

$$
\hat{\psi}\left(x^{\prime}\right)=\left(\hat{f}_{1} \circ \varphi\left(x^{\prime}\right), \ldots, \hat{f}_{p} \circ \varphi\left(x^{\prime}\right)\right)
$$

derivando

$$
\begin{aligned}
D_{x^{\prime}} \hat{\psi}(0)= & D_{x^{\prime}} \hat{f}_{i} \circ \varphi\left(x^{\prime}\right)=D_{x^{\prime}} \hat{f}_{i} \circ \varphi\left(x^{\prime}\right) . D_{x^{\prime}} \varphi\left(x^{\prime}\right) \\
= & {\left[\begin{array}{cccc}
\frac{\partial f_{1}}{\partial x_{1}} \varphi(0) & \ldots & \frac{\partial f_{1}}{\partial x_{p}} \varphi(0) & \\
\vdots & & & 0 \\
\frac{\partial f_{p}}{\partial x_{1}} \varphi(0) & \ldots & \frac{\partial f_{p}}{\partial x_{p}} \varphi(0)
\end{array}\right]_{p \times n}\left[\begin{array}{cccc}
1 & 0 & \ldots & 0 \\
0 & 1 & \ldots & 0 \\
\vdots & & & \\
0 & \ldots & 0 & 1 \\
& & 0 &
\end{array}\right]_{n \times p} } \\
= & {\left[\begin{array}{ccc}
\frac{\partial f_{1}}{\partial x_{1}} \varphi(0) & \ldots & \frac{\partial f_{1}}{\partial x_{p}} \varphi(0) \\
\vdots & & \\
\frac{\partial f_{p}}{\partial x_{1}} \varphi(0) & \ldots & \frac{\partial f_{p}}{\partial x_{p}} \varphi(0)
\end{array}\right]_{p \times p}=I }
\end{aligned}
$$

es decir, existe $\hat{\varphi} \in \mathbb{K}\left[\left[x^{\prime}\right]\right]^{p \times p}$ tal que $\hat{\varphi} \circ \hat{\psi}\left(x^{\prime}\right)=x^{\prime}$, donde $\hat{\varphi}=\left(\hat{\varphi}_{1}, \ldots, \hat{\varphi}_{p}\right)$, los $\hat{h}_{i}(x)$ poseen las mismas propiedades que los $\hat{f}_{i}$ puesto que

$$
\begin{aligned}
\hat{h}_{i}(x) & =\hat{\varphi}_{i}\left(\hat{f}_{1}(x), \ldots, \hat{f}_{p}(x)\right)=\hat{\varphi}_{i}\left(\hat{f}_{1}\left(x^{\prime}, 0\right), \ldots, \hat{f}_{p}\left(x^{\prime}, 0\right)\right) \\
& =\hat{\varphi}_{i}\left(\hat{\psi}_{1}\left(x^{\prime}\right), \hat{\psi}_{2}\left(x^{\prime}\right), \ldots, \hat{\psi}_{p}\left(x^{\prime}\right)\right) \\
& =\hat{\varphi}_{i} \hat{\psi}\left(x^{\prime}\right) \\
& =x^{\prime}
\end{aligned}
$$

$\hat{h}_{i}\left(x^{\prime}, 0\right)=x_{i}$ y $\omega_{1} \wedge \omega_{2} \wedge \ldots \wedge \omega_{p} \wedge d \hat{h}_{i}=0$, en efecto,

$$
\begin{aligned}
\frac{\partial \hat{h}_{i}}{\partial x_{i}} & =\sum_{j=1}^{p} \frac{\partial \hat{\varphi}_{i}}{\partial y_{j}} \frac{\partial \hat{f}_{j}}{\partial x_{i}} \\
d \hat{h}_{i} & =\sum_{i=1}^{p} \frac{\partial \hat{h}_{i}}{\partial x_{i}} d x_{i} \\
& =\sum_{i=1}^{p}\left(\sum_{j=1}^{p} \frac{\partial \hat{\varphi}_{i}}{\partial y_{j}} \frac{\partial \hat{f}_{j}}{\partial x_{i}}\right) d x_{i} \\
& =\sum_{j=1}^{p} \frac{\partial \hat{\varphi}_{i}}{\partial y_{j}} \sum_{i=1}^{p} \frac{\partial \hat{f}_{j}}{\partial x_{i}} d x_{i}=\sum_{j=1}^{p} \frac{\partial \hat{\varphi}_{i}}{\partial y_{j}} d f_{j}
\end{aligned}
$$

Luego,

$$
\begin{aligned}
\omega_{1} \wedge \ldots \wedge \omega_{p} \wedge d \hat{h}_{i} & =\omega_{1} \wedge \ldots \wedge \omega_{p} \wedge \sum_{j=1}^{p} \frac{\partial \hat{\varphi}_{i}}{\partial y_{j}} d f_{j} \\
& =\sum_{j=1}^{p} \frac{\partial \hat{\varphi}_{i}}{\partial y_{j}} \omega_{1} \wedge \ldots \wedge \omega_{p} \wedge d f_{j} \\
& =0
\end{aligned}
$$


Las integrales primeras naturales $h_{i}$ de $\omega_{1}, \omega_{2}, \ldots, \omega_{p}$ verifican igualmente:

$$
h_{i}\left(x^{\prime}, 0\right)=x_{i}, \omega_{1} \wedge \ldots \wedge \omega_{p} \wedge d h_{i}=0
$$

luego para $i=1, \ldots, p$ :

$$
h_{i}\left(x^{\prime}, 0\right)=\hat{h}_{i}\left(x^{\prime}, 0\right)=x_{i}, \quad d h_{1} \wedge d h_{2} \wedge \ldots \wedge d h_{p} \wedge d \hat{h}_{i}=0 .
$$

Del teorema de funciones compuestas $\left([7]\right.$, p. 1237) en $\mathbb{K}[[x]]^{p \times p}$ tenemos que existen $\widehat{\Psi}_{i} \in \mathbb{K}\left[\left[x^{\prime}\right]\right]^{p \times p}$ tales que:

$$
\widehat{h}_{i}(x)=\widehat{\Psi}_{i}\left(h_{1}(x), h_{2}(x), \ldots, h_{p}(x)\right) \text { para } i=1, \ldots, p .
$$

Puesto que $h_{i}\left(x^{\prime}, 0\right)=\hat{h}_{i}\left(x^{\prime}, 0\right)$ obtenemos $\left(\widehat{\Psi}_{1}, \widehat{\Psi}_{2}, \ldots, \widehat{\Psi}_{p}\right)=\mathrm{I}$ y $h_{i}=\hat{h}_{i}$ para $i=1, \ldots, p$.

\section{Blowing-up}

Espacio Proyectivo Un espacio proyectivo de dimensión $n$ sobre un campo $\mathbb{K}$ es un conjunto $\mathbb{P}$ con una aplicación exhaustiva $\pi: F-\{0\} \longrightarrow \mathbb{P}$, donde $F$ es un $\mathbb{K}$-espacio vectorial $(n+1)$ dimensional y $\pi(v)=\pi(w)$ si y solo si $v=\lambda w$ para algún $\lambda \in \mathbb{K}$. A menudo decimos que $\pi$ define una estructura de espacio proyectivo en $\mathbb{P}$. Una vez que se ha fijado una base en $F$ las componentes de un vector $v$ no nulo serán tomadas como las coordenadas homogéneas de $\pi(v)$, las coordenadas homogéneas de un punto siendo determinadas por una constante multiplicativa. Usualmente se denota por $\left[x_{0}: \cdots: x_{n}\right]$ para el punto de coordenadas homogéneas $x_{0}, \ldots, x_{n}$.

Si $\mathbb{P}_{1}$ es un espacio proyectivo unidimensional y $z_{0}, z_{1}$ son coordenadas proyectivas en $\mathbb{P}_{1}$ la aplicación inyectiva

$$
\begin{aligned}
\mathbb{P}_{1} & \longrightarrow \mathbb{C} \cup\{\infty\} \\
{\left[z_{0}: z_{1}\right] } & \longmapsto \frac{z_{0}}{z_{1}}
\end{aligned}
$$

asumimos que envía $[1: 0]$ en $\infty$, será llamada una coordenada absoluta en $\mathbb{P}_{1}$.

Singularidad Simple Sea $\mathcal{F}$ un germen de foliación en $\left(\mathbb{C}^{2}, 0\right)$ y $\omega=a(x, y) d x+b(x, y) d y$ un generador de $\mathcal{F}$. Supongamos que el origen es un punto singular de $\mathcal{F}$. Podemos decir que el origen es una singularidad simple de $\mathcal{F}$ si la matriz

$$
J_{0}(\omega ; x, y)=\left(\begin{array}{cc}
-\frac{\partial b}{\partial x}(0) & \frac{\partial a}{\partial x}(0) \\
-\frac{\partial b}{\partial y}(0) & \frac{\partial a}{\partial y}(0)
\end{array}\right)
$$

tiene dos valores propios $\lambda \neq \mu, \mu \neq 0$, tales que $\frac{\lambda}{\mu} \notin \mathbb{Q}^{+}$.

La definición no depende de la elección de $\omega$ ni de las coordenadas elegidas.

Una vez que un punto 0 sobre una superficie (suave) $M$ es fijado, escogemos coordenadas locales $x, y$ en 0 y asumimos que ellos son analíticas en una vecindad abierta $U$ de 0 . Tomamos una recta proyectiva compleja $\mathbb{P}_{1}$, con coordenadas homogéneas $z_{0}, z_{1}$ y sea $\bar{U}$ una subvariedad de $U \times \mathbb{P}_{1}$ definida por la ecuación $x z_{1}-y z_{0}=0$. Sean las rectas afines $\mathbb{A}_{1}: z_{0} \neq 0$ y $\mathbb{A}_{1}^{\prime}: z_{1} \neq 0$, con coordenadas $z=z_{1} / z_{0}$ y $z^{\prime}=z_{0} / z_{1}$, respectivamente, un cubrimiento abierto de $\mathbb{P}_{1}$; entonces tenemos $U \times \mathbb{P}_{1}=U \times \mathbb{A}_{1} \cup U \times \mathbb{A}_{1}^{\prime}$ y el trazo de $\bar{U}$ en cada pieza $U \times \mathbb{A}_{1}$ y $U \times \mathbb{A}_{1}^{\prime}$ es definido por las ecuaciones

$$
x z-y=0 \quad \text { y } \quad x-y z^{\prime}=0
$$

respectivamente. Podemos establecer: 
Lema 5.1 $\bar{U}$ es una superficie(suave y conexa). La proyección $U \times \mathbb{P}_{1} \longrightarrow U$ induce un morfismo analítico $\pi: \bar{U} \rightarrow U$ cuya restricción a $\bar{U}-\pi^{-1}(0)$ es un isomorfismo sobre $U-\{0\}$.

Demostración. Ponemos $V=\bar{U} \cap U \times \mathbb{A}_{1}$ y $V^{\prime}=\bar{U} \cap U \times \mathbb{A}_{1}^{\prime}$. Que $\bar{U}$ sea una superficie suave y conexa se desprende de las ecuaciones para $V$ y $V^{\prime}$ dadas anteriormente. Para el resto es suficiente decir que los morfismos

$$
\begin{aligned}
U-\{x=0\} & \longrightarrow V \\
(x, y) & \longmapsto(x, y, y / x)
\end{aligned}
$$

$\mathrm{y}$

$$
\begin{aligned}
U-\{y=0\} & \longrightarrow V^{\prime} \\
(x, y) & \longmapsto(x, y, x / y)
\end{aligned}
$$

uniendolos para dar la inversa y obtener el isomorfismo pedido.

Observación 5.1 Ya que en el conjunto abierto $V$ tenemos $y=x z$, es claro que $x, z$ pueden ser tomados como coordenadas analíticas en el conjunto $V$. Similarmente, $y, z^{\prime}$ son coordenadas analíticas en el conjunto $V^{\prime}$ dados por $(x, z) \longmapsto(x, z x)$ y $\left(y, z^{\prime}\right) \longmapsto\left(y z^{\prime}, y\right)$, y las coordenadas de un punto en ambos $V$ y $V^{\prime}$ están relacionados por $y=z x, z^{\prime}=1 / z$.

El morfismo $\pi: \bar{U} \longrightarrow U$ que acabamos de definir, o más bien su correspondencia inversa, tenemos en 0 el efecto local que queremos. Todo lo que necesitamos es extenderlo a todo $M$, lo cual no es difícil puesto que $\pi_{\bar{U}-\pi^{-1}(0)}$ es un isomorfismo.

Notemos primero que la gráfica $\pi_{\bar{U}-\pi^{-1}(0)}$ es igual a la traza en $\bar{U} \times(M-\{0\})$ de $\left\{(p, q, p) \mid p \in M, q \in \mathbb{P}_{1}\right\}$, claramente un subconjunto cerrado de $M \times \mathbb{P}_{1} \times M$.

Luego la gráfica de $\pi_{\bar{U}-\pi^{-1}(0)}$ es cerrada en $\bar{U} \times(M-\{0\})$ y podemos definir:

Definición 5.1 Sea $\bar{M}$ la superficie obtenida de la unión de $\bar{U}$ y $M-\{0\}$ por el isomorfismo

$$
\pi_{\bar{U}-\pi^{-1}(0)}: \bar{U} \times(M-\{0\}) \simeq U-\{0\}
$$

y aún denotaremos por $\pi$ al morfismo $\pi$ extendido por la identidad en $M-\{0\}$ así $\pi: \bar{M} \longrightarrow M$ es llamada el blowing-up de 0 en $M$. También decimos que $\bar{M}$ es obtenido $M$ por blowing-up del punto 0 y que 0 es el centro del blowing-up.

Es claro que, después del blowing-up $0, M-\{0\}$ esencialmente no fueron modificados mientras que el correspondiente a 0 en $\bar{H}$ es una recta proyectiva, decimos $E=\pi^{-1}(0)=\{0\} \times \mathbb{P}_{1}$. Tal recta $E$ es llamada el divisor excepcional de $\pi$. La restricción de $\pi$,

$$
\left.\pi\right|_{\bar{M}-E}: \bar{M}-E \longrightarrow M-\{0\}
$$

es un isomorfismo. se puede notar que el divisor excepcional $E$ está contenido en $V \cup V^{\prime}$ y las ecuaciones $x=0$ en $V$ e $y=0$ en $V^{\prime}$. Esto en particular muestra que $E$ es suave en todos sus puntos y por esto es una subvariedad unidimensional de $\bar{M}$, claramente isomorfo a una recta proyectiva compleja y por eso es compacto y conexo. Note también que $\bar{M}-E$ es denso en $\bar{M}$, lo cual será muy útil. La siguiente proposición nos muestra que la explosión de 0 en $H$ no depende del subconjunto abierto $U$ y las coordenadas locales $x, y$ que hemos usado en la definición.

Proposición 5.1 Sea $\pi^{\prime}: \bar{M}^{\prime} \longrightarrow M$ una segunda explosión de 0 en $M$, obtenida de una vecindad abierta $U^{\prime}$ de 0 y coordenadas $x^{\prime}, y^{\prime}$ en él. Entonces existe un único $M$-isomorfismo $\varphi: \bar{M} \longrightarrow \bar{M}^{\prime}$. Además $\varphi$ restricta a la recta proyectiva entre $E$ y $E^{\prime}=\pi^{\prime-1}(0)$. 
Demostración. Desde que ambas $x, y$ y $x^{\prime}, y^{\prime}$ son coordenadas locales en 0 , podemos determinar una tercera vecindad abierta de $0, U^{\prime \prime} \subset U \cap U^{\prime}$, y funciones analíticas definidas en $U^{\prime \prime}$, $a_{i, j}, i, j=0,1$, tales que

$$
\begin{aligned}
x^{\prime} & =a_{0,0} x+a_{0,1} y \\
y^{\prime} & =a_{1,0} x+a_{1,1} y
\end{aligned}
$$

$\mathrm{y} \operatorname{det}\left(a_{i, j}(p)\right) \neq 0$ para todo $p \in U^{\prime \prime}$. entonces es fácil ver que

$$
\begin{aligned}
U^{\prime \prime} \times \mathbb{P}_{1} & \longrightarrow U^{\prime \prime} \times \mathbb{P}_{1} \\
\left(p, z_{0}, z_{1}\right) & \longmapsto\left(p, a_{0,0} z_{0}+a_{0,1} z_{1}, a_{1,0} z_{0}+a_{1,1} z_{1}\right)
\end{aligned}
$$

es un $U^{\prime \prime}$-isomorfismo que restricto a un $U^{\prime \prime}$-isomorfismo $\psi$ de $\pi^{-1}\left(U^{\prime \prime}\right)$ en $\pi^{\prime-1}\left(U^{\prime \prime}\right)$. Así $\varphi$ es obtenida al extender $\psi$ a $\bar{M}$ usando $\left.\left(\left.\pi^{\prime}\right|_{\bar{M}^{\prime}-E^{\prime}}\right)^{-1}{ }_{\circ} \pi\right|_{\bar{M}-E}$.

Para la unicidad de $\varphi$ es suficiente recordar la condición que determina el $M$-isomorfismo $\varphi$ en $\bar{M}-E$, el cual es un subconjunto denso de $\bar{M}$.

Por último es claro de la definición de $\varphi$ que $\left.\varphi\right|_{E}$ es una recta proyectiva.

Corolario 5.2 Supongamos que $\pi: \bar{M} \longrightarrow M$ y $\hat{\pi}: \bar{M}^{\prime} \longrightarrow M^{\prime}$ son las explosiones de los puntos 0 y $0^{\prime}$ en $M$ y $M^{\prime}$, respectivamente. Si $\varphi: W \longrightarrow W^{\prime}$ es un isomorfismo analítico entre vecindades de 0 y $0^{\prime}$ y $\varphi(0)=0^{\prime}$, entonces existe un único isomorfismo $\bar{\varphi}: \pi^{-1}(W) \longrightarrow \hat{\pi}^{-1}\left(W^{\prime}\right)$ tales que $\left.\varphi \circ \pi\right|_{\pi^{-1}(W)}=\hat{\pi}_{\circ} \bar{\varphi}$. Además $\bar{\varphi}$ restricta a una recta proyectiva entre los divisores excepcionales $E=\pi^{-1}(0)$ y $E^{\prime}=\hat{\pi}^{-1}\left(0^{\prime}\right)$.

Demostración. La condición $\left.\varphi \circ \pi\right|_{\pi^{-1}(W)}=\hat{\pi}_{\circ} \bar{\varphi}$. determina $\bar{\varphi}$ en $\pi^{-1}(W)-E$ es denso en $\pi^{-1}(W)$.

Para la existencia de $\bar{\varphi}$, sean $x, y$ coordenadas locales en una vecindad abierta $U \subset W$ de 0 , y tomamos $x \circ \varphi^{-1}$ e $y \circ \varphi^{-1}$ como coordenadas locales en $U^{\prime}=\varphi(U)$.

Usando tales coordenadas para construir las explosiones, es claro que $\varphi \times$ Id $: U \times \mathbb{P}_{1} \longrightarrow U^{\prime} \times \mathbb{P}_{1}$ restricta a un isomorfismo $\pi^{-1}(U) \simeq \hat{\pi}^{-1}\left(U^{\prime}\right)$ cuya extensión obvia a $\pi^{-1}(W)$ es $\bar{\varphi}$. La última parte se sigue de la definición de $\bar{\varphi}$ dada.

Observación 5.2 En particular del corolario 5.2 se sigue que para cualquier vecindad abierta $W$ de 0 en $M, \pi^{-1}(W)$ puede ser canónicamente identificado con la superficie $\bar{W}$ obtenida de $W$ por la explosión en 0 .

\subsection{Transformando Curvas}

Sea $\pi: \bar{M} \longrightarrow M$ la explosión de 0 en $M$ y sea $x, y$, como antes, un sistema de coordenadas locales en 0. En adelante, para evitar confusiones, si $f$ es una función analítica definida en algún conjunto abierto $U \subset M$, denotemos por $\bar{f}$ a la función $f$ 。 $\pi$ la cual es analítica en $\pi^{-1}(U)$. Esto aplicado en particular a las funciones $x \circ \pi$ e $y \circ \pi$ que hasta ahora hemos denotado por $x$ e $y$.

Sea $\xi$ una curva en un subconjunto abierto $W$ de $M$ : denotaremos por $\bar{\xi}$ al pull-back de $\xi$ por $\pi, \bar{\xi}=\pi^{*}(\xi)$, y lo llamaremos el transformado total de $\xi$ (después de la explosión en 0 ). Como es de esperarse, si $0 \in \xi$, entonces $E=\pi^{-1}(0)$ necesita estar contenida en $\bar{\xi}=\pi^{*}(\xi)$. Más precisamente:

Lema 5.3 El transformado total de $\xi$ tiene la forma

$$
\bar{\xi}=\tilde{\xi}+\operatorname{mult}(\xi) E
$$

donde $\tilde{\xi}$ es una curva en $\pi^{-1}(W)$ con un número finito de intersecciones con $E$. 
Demostración. Supongamos que $x, y$ son coordenadas locales en 0 y $f$ una ecuación de $\xi$, todas definidas en una vecindad $U$ de 0 . Consideremos, como en la sección anterior, $\pi^{-1}(U)$ cubierta por subconjuntos abiertos $V: \bar{x} \neq 0$ y $V^{\prime}: \bar{y} \neq 0$. Escribimos $f=f_{e}+f_{e+1}+\cdots+f_{i}+\cdots$, donde $e=$ mult $(\xi)$ y $\operatorname{los} f_{i}$ son formas de grado $i$ en las coordenadas locales $x, y$. Usando en $V$ las coordenadas $\bar{x}$ y $z=\bar{y} / \bar{x}$, tenemos las ecuaciones de $\bar{\xi}$ en $V$ :

$$
\begin{aligned}
\bar{f} & =f_{e}(\bar{x}, \bar{x} z)+f_{e+1}(\bar{x}, \bar{x} z)+\cdots+f_{i}(\bar{x}, \bar{x} z)+\cdots \\
& =\bar{x}^{e} f_{e}(1, z)+\bar{x}^{e+1} f_{e+1}(1, z)+\cdots+\bar{x}^{i} f_{i}(1, z)+\cdots .
\end{aligned}
$$

Luego $\tilde{f}=\bar{f} / x^{e}$ define una curva en $V$ con un número finito de intersecciones con $E$, es decir, los puntos $(0, z)$ para los cuales $f_{e}(1, z)=0$. Un cálculo similar muestra que también $y^{e}$ divide $\bar{f}$ en $V^{\prime}$ y $\tilde{f}^{\prime}=\bar{f} / y^{e}$ define en $V^{\prime}$ una curva con un número finito de intersecciones con $E$. Desde que $\tilde{f} / \tilde{f}^{\prime}=y^{e} / x^{e}$ no tienen ceros en $V \cap V^{\prime}$, estas curvas unidas dan una curva en $\pi^{-1}(U)$, la cual puede ser extendida a la curva $\tilde{\xi}$ deseada en el conjunto $\pi^{-1}(W)$ usando las ecuaciones locales de $\tilde{\xi}$ en $\pi^{-1} W-E$.

\section{Criterio de convergencia de Mattei - Moussu}

En esta sección veremos la prueba en el campo de los números complejos del teorema de Malgrange, veamos algunos resultados.

\subsection{Transversalidad}

Sea $\mathcal{F}$ un germen de foliaciones en el origen de $\mathbb{C}^{n}$. Un germen de inmersión $i:\left(\mathbb{C}^{p}, 0\right) \rightarrow$ $\left(\mathbb{C}^{n}, 0\right)$ es transversal a $\mathcal{F}$ si

$$
\left\{\begin{array}{l}
\operatorname{Sing}\left(i^{-1} \mathcal{F}\right)=i^{-1} \operatorname{Sing} \mathcal{F} \\
\operatorname{Codim}\left(\operatorname{Sing}\left(i^{-1} \mathcal{F}\right)\right)=\inf (p, \operatorname{Codim}(\mathcal{F}))
\end{array}\right.
$$

Cuando $p=2$, la inmersión $i:\left(\mathbb{C}^{2}, 0\right) \rightarrow\left(\mathbb{C}^{n}, 0\right)$ es transversal a $\mathcal{F}$ si y sólo si $\operatorname{Sing}\left(i^{-1} \mathcal{F}\right)=\{0\}$. Aquí describiremos bajo que condiciones una inmersión lineal general de $\mathbb{C}^{2}$ en $\mathbb{C}^{n}$ es transversal a $\mathcal{F}$ (en el origen).

Sea $\omega=\sum_{i=1}^{n} a_{i} d x_{i}$ una 1 -forma definiendo $\mathcal{F}$. Supongamos que el coeficiente $a_{1}$ no es idénticamente nulo (siempre es posible suponer esta condición mediante un cambio de coordenadas lineales). Sea $B \in \mathbb{C}^{n-2}$, con $B=\left(B_{3}, \ldots, B_{n}\right)$ consideremos la inmersión lineal

$$
i_{B}:\left(x_{1}, x_{2}\right) \mapsto\left(x_{1}, x_{2}, B_{3} x_{2}, \ldots, B_{n} x_{2}\right)
$$

Tenemos

$$
\begin{aligned}
i_{B}^{*}= & a_{1}\left(x_{1}, x_{2}, B_{3} x_{2}, \ldots, B_{n} x_{2}\right) d x_{1}+a_{2}\left(x_{1}, x_{2}, B_{3} x_{2}, \ldots, B_{n} x_{2}\right)+ \\
& a_{3}\left(x_{1}, x_{2}, B_{3} x_{2}, \ldots, B_{n} x_{2}\right) B_{3} d x_{2}+\cdots+a_{n}\left(x_{1}, x_{2}, B_{3} x_{2}, \ldots, B_{n} x_{2}\right) B_{n} d x_{2} \\
= & a_{1}\left(x_{1}, x_{2}, B_{3} x_{2}, \ldots, B_{n} x_{2}\right) d x_{1}+ \\
& +\left[a_{2}\left(x_{1}, x_{2}, B_{3} x_{2}, \ldots, B_{n} x_{2}\right)+\sum_{i=3}^{n} B_{i} a_{i}\left(x_{1}, x_{2}, B_{3} x_{2}, \ldots, B_{n} x_{2}\right)\right] d x_{2}
\end{aligned}
$$

e $i_{B}$ no es transversal a $\mathcal{F}$ si y solamente si las dos curvas $a_{1}\left(x_{1}, x_{2}, B_{3} x_{2}, \ldots, B_{n} x_{2}\right)=0$ $a_{2}\left(x_{1}, x_{2}, B_{3} x_{2}, \ldots, B_{n} x_{2}\right)+\sum_{i=3}^{n} B_{i} a_{i}\left(x_{1}, x_{2}, B_{3} x_{2}, \ldots, B_{n} x_{2}\right)=0$ tienen una componente en común. Se verifica que cada $B \in \mathbb{C}^{n-2}$, las hipersuperficies de $\mathbb{C}^{n}$

$$
a_{1}=0 \text { у } a_{2} x_{2}+\cdots+a_{n} x_{n}=0
$$


tienen una componente en común.

Introducimos la función $P_{\omega}(x)=\sum x_{i} a_{i}(x)$ ella no depende del sistema lineal de coordenadas elegido, en ese sentido si $\sigma: \mathbb{C}^{n} \rightarrow \mathbb{C}^{n}$ es un isomorfismo lineal, tenemos

$$
P_{\sigma^{*} \omega}=P_{\omega} \circ \sigma
$$

Se verifica que $i_{B}$ no es transversal a $\mathcal{F}$ para todo $B$ si y sólo si $a_{1}$ y $P_{\omega}$ tienen una componente en común.

Lema 6.1 En la situación anterior, supongamos que $P_{\omega} \not \equiv 0$. Existe un isomorfismo lineal $\sigma: \mathbb{C}^{n} \rightarrow \mathbb{C}^{n}$ tal que si:

$$
\sigma^{*} \omega=a_{1}^{(\sigma)} d x_{1}+\cdots+a_{n}^{(\sigma)} d x_{n}
$$

entonces $a_{1}^{(\sigma)}$ y $P_{\sigma^{*} \omega}$ no tienen componentes en común. Además, uno puedo escoger $\sigma$ de la forma

$$
\sigma_{\alpha}\left(x_{1}, \ldots, x_{n}\right)=\left(x_{1}, x_{2}+\alpha_{2} x_{1}, \ldots, x_{n}+\alpha_{n} x_{1}\right)
$$

$\operatorname{para} \alpha \in \mathbb{C}^{n-1}$.

Demostración. Si $a_{1}^{(\sigma)}$ y $P_{\sigma^{*} \omega}$ tienen una rama en común para todo $\alpha$ entonces $a_{1}^{(\sigma)} \circ \sigma_{\alpha}^{-1}$ y $P_{\omega}$ tienen una rama común para todo $\alpha$, es decir,

$$
\begin{aligned}
a_{1}^{(\sigma)} & =h P_{\sigma^{*} \omega} \\
a_{1}^{(\sigma)} \circ \sigma_{\alpha}^{-1} & =h P_{\sigma^{*} \omega} \circ \sigma_{\alpha}^{-1} \\
& =h P_{\omega} \circ \sigma \circ \sigma_{\alpha}^{-1} \\
& =h P_{\omega}
\end{aligned}
$$

Pero

$$
\begin{aligned}
a_{1}^{(\sigma)} \circ \sigma_{\alpha}^{-1}\left(x_{1}, \ldots, x_{n}\right) & =h P_{\omega}\left(x_{1}, \ldots, x_{n}\right) \\
a_{1}^{(\sigma)}\left(x_{1}, x_{2}-\alpha_{2} x_{1}, \ldots, x_{n}-\alpha_{n} x_{1}\right) & =h \sum x_{i} a_{i}(x) \\
& =h \circ\left(x_{1} a_{1}+\left(x_{2}-\alpha_{2} x_{1}\right) a_{2}+\cdots+\left(x_{n}-\alpha_{n} x_{1}\right) a_{n}\right)(x) \\
& =h \circ\left(x_{1}\left(a_{1}-\alpha_{2} a_{2}-\cdots-\alpha_{n} a_{n}\right)+x_{2} a_{2}+\cdots+x_{n} a_{n}\right)(x) \\
& =h \circ\left(x_{1}\left(a_{1}-\alpha_{2} a_{2}-\cdots-\alpha_{n} a_{n}\right)\right)(x) \\
& =a_{1}-\alpha_{2} a_{2}-\cdots-\alpha_{n} a_{n} \\
& =a_{1}+\sum_{i \geq 2} \alpha_{i} a_{i}
\end{aligned}
$$

Sea $P_{1}^{r_{1}} \ldots P_{q}^{r_{q}}$ la descomposición en componentes irreducibles de $P_{\omega}$. Denotemos

$$
U_{j}=\left\{\alpha \in \mathbb{C}^{n-1} ; a_{1}^{(\sigma)} \circ \sigma_{\alpha}^{-1} \text { es divisible por } P_{j}\right\}
$$

Los $U_{j}$ son cerrados analíticos de $\mathbb{C}^{n-1}$ que recubren todo $\mathbb{C}^{n-1}$. Pues si hay uno, digamos un $U_{k}$, que coincide con $\mathbb{C}^{n-1}$. Deducimos que $P_{k}$ divide $a_{1}+\sum_{i \geq 2} \alpha_{i} a_{i}$ para todo $\alpha$ y para cada $a_{j}$, se tiene un absurdo.

Supondremos que $P_{\omega} \equiv 0$. esto significa que $\mathcal{F}$ es proyectiva y se permite definir una 1 -forma homogénea $W$. Un cálculo elemental nos muestra que un cambio "cuadrático" genérico

$$
\sigma_{Q}: x \mapsto x+Q(x)
$$

produce $P_{\sigma_{Q}^{*} W} \not \equiv 0$. Obtenemos luego el siguiente teorema. 
Teorema 6.1 Sea $\mathcal{F}$ un germen de foliación en el origen de $\mathbb{C}^{n}$ definido en coordenadas lineales por la 1-forma $\omega=\sum_{i=1}^{n} a_{i} d x_{i}$ y consideremos la función $P_{\omega}(x)=\sum x_{i} a_{i}$. Entonces tenemos

1. Si $P_{\omega}(x) \not \equiv 0$, el conjunto de inmersiones lineales $i: \mathbb{C}^{2} \rightarrow \mathbb{C}^{n}$ transversal a $\mathcal{F}$ es un abierto de Zariski no vacío.

2. Si $P_{\omega}(x) \equiv 0$, el conjunto de inmersiones "cuadráticas" $i: \mathbb{C}^{2} \rightarrow \mathbb{C}^{n}$ transversal a $\mathcal{F}$ es un abierto de Zariski no vacío.

Mostraremos que un germen de foliaciones en $\mathbb{C}^{2}$ que posee una integral primera formal posee una convergente, para cierto tipo de integrales primeras. Desarrollaremos el caso de singularidades simples y obtendremos el caso general por inducción a lo largo de la desingularización.

Lema 6.2 Sea $\mathcal{F}$ un germen de foliaciones con una singularidad simple en el origen de $\mathbb{C}^{2}$. Si $\mathcal{F}$ tiene una integral primera formal no constante $\hat{f}$, entonces $\mathcal{F}$ tiene una integral primera holomorfa $f$.

Demostración. Como las componentes irreducibles de $\hat{f}$ son curvas integrales formales y $\mathcal{F}$ tiene una singularidad simple en el origen, existe un sistema de coordenadas formales $(\hat{x}, \hat{y})$ tal que

$$
\hat{f}=\hat{x}^{p} \hat{y}^{q}
$$

donde $p$ y $q$ son enteros primos entre ellos. Por consiguiente, $\mathcal{F}$ es formalmente linealizable y es dado por

$$
\hat{x} \hat{y}\left(p \frac{d \hat{x}}{\hat{x}}+q \frac{d \hat{y}}{\hat{y}}\right)
$$

El teorema de Briot-Bouquet ([?], p. 166) asegura la convergencia de las curvas $\hat{x}=0$ e $\hat{y}=0$. Así, existe un sistema de coordenadas convergentes $(x, y)$ en el que $\mathcal{F}$ está definido por

$$
\omega=p y d x+q x(1+U(x, y)) d y, \quad U(0)=0
$$

Se sigue que

$$
\hat{f}(x, y)=x^{p} y^{q} \widehat{W}(x, y)=\hat{x}^{p} \hat{y}^{q}
$$

donde $\widehat{W}(x, y)$ es una unidad formal $\widehat{W}(0)=1$. Consideremos el campo de vectores (que es definido por el germen de la foliación $\mathcal{F}$ )

$$
X=q x(1+U) \frac{\partial}{\partial x}-p y \frac{\partial}{\partial y}
$$

y sea $\phi_{t}(x, y)=\exp t X$ el grupo con un parámetro de $X$. Tenemos

$$
\phi_{t}(x, y)=\left(x e^{q t} V(x, y, t), y e^{-p t}\right), V(0,0, t)=V(x, y, 0)=1
$$

Supongamos $X$ definido en una bola $B \subset \mathbb{C}^{2}$ centrada en el origen. Si $b$ es lo suficientemente pequeño, la aplicación $(x, y, t) \mapsto \phi_{t}(x, y)$ está definida en $B \times D(0,1)$, donde $D(0,1) \subset \mathbb{C}$ es el disco centrado en el origen y de radio uno. Como $X f=0$, tenemos que $\hat{f} \circ \phi_{t}=\hat{f}$ para todo $t \in D(0,1)$. En particular $\hat{f} \circ \phi_{2 i \pi}=\hat{f}$

El difeomorfismo $\phi_{2 i \pi}(x, y)=(x V(x, y, 2 i \pi), y)=\left(x V_{1}(x, y), y\right)$ es tangente a la identidad y por lo anterior

$$
V_{1}^{p}(x, y) \widehat{W}\left(x V_{1}, y\right)=\widehat{W}(x, y)
$$


El teorema de la función implícita, aplicada a la ecuación anterior nos conduce a $V_{1} \equiv 1 \mathrm{y}$ $\phi_{2 i \pi}=\operatorname{Id}_{\mathbb{C}^{2}}$. En efecto,

$$
\begin{aligned}
V_{1}(0,0) & =1 \\
F(x, y, t) & =z^{p} \widehat{W}(x z, y)-\widehat{W}(x, y)=0, \quad F(0,0,1)=0 \\
\frac{\partial F}{\partial z}(x, y, z) & =p z^{p-1} \widehat{W}(x z, y)-z^{p} \frac{\partial \widehat{W}}{\partial x}(x z, y) z \\
\frac{\partial F}{\partial z}(0,0,1) & =p \widehat{W}(0,0) \neq 0
\end{aligned}
$$

Notemos que $D \phi_{t}(0)$ la diferencial de $\phi_{t}$ en el punto 0 e introducimos la aplicación

$$
H(x, y)=\int_{0}^{1}\left[D \phi_{2 i \pi t}(0)\right]^{-1} \phi_{2 i \pi t}(x, y) d t
$$

que es holomorfa en una vecindad del origen de $\mathbb{C}^{2}$ y $D H(0)=\mathrm{Id}$. Para un número real $s$ cercano al 0, tenemos:

$$
\begin{aligned}
H \circ \phi_{2 i \pi s}(x, y) & =\int_{0}^{1}\left[D \phi_{2 i \pi t}(0)\right]^{-1} \phi_{2 i \pi(t+s)}(x, y) d t \\
& =\int_{0}^{1} D \phi_{2 i \pi s}(0)\left[D \phi_{2 i \pi(t+s)}(0)\right]^{-1} \phi_{2 i \pi(t+s)}(x, y) d t \\
& =D \phi_{2 i \pi s}(0) \int_{0}^{1}\left[D \phi_{2 i \pi(t+s)}(0)\right]^{-1} \phi_{2 i \pi(t+s)}(x, y) d t
\end{aligned}
$$

Como $t \mapsto \phi_{2 i \pi t}$ es periódica de periodo 1, podemos escribir

$$
\int_{0}^{1}\left[D \phi_{2 i \pi(t+s)}(0)\right]^{-1} \phi_{2 i \pi(t+s)}(x, y) d t=\int_{0}^{1}\left[D \phi_{2 i \pi t}(0)\right]^{-1} \phi_{2 i \pi t}(x, y) d t=H(x, y)
$$

Finalmente $H \circ \phi_{2 i \pi s}(x, y)=\left(D \phi_{2 i \pi s}(0) \circ H\right)(x, y)$ para todo $s$ suficientemente pequeño y como $H$ es holomorfo, tenemos que

$$
H \circ \phi_{t}=D \phi_{t}(0) \circ H .
$$

De esto decimos que $H$ linealiza $X$. El campo $X$ y luego $\omega$, posee una integral primera convergente $f$, que evidentemente se puede elegir de la forma $x^{p} y^{q}$.

Observación 6.1 Sea $f=x^{p} y^{q}$ como se desea. Entonces, $\hat{g}$ es una integral primera formal de $\mathcal{F}$, si existe $\hat{\tau} \in \mathbb{C}[[t]]$ tal que $\hat{g}=\hat{\tau} \circ f$. Además $\hat{g}$ es convergente si y solamente si $\hat{\tau}$ es convergente.

El caso general Lo obtenemos a partir del caso de singularidades simples y del comportamiento de las integrales primeras por explosiones.

Lema 6.3 Sea $\widehat{F} \in \mathbb{C}\left[\left[x_{1}, \ldots, x_{n}\right]\right]$ y $\pi: N^{\prime} \rightarrow\left(\mathbb{C}^{n}, 0\right)$ la explosión del origen en $\mathbb{C}^{n}$. Si existe un punto $P \in \pi^{-1}(0)$ tal que $(\widehat{F} \circ \pi)_{P}$ es convergente, entonces $\widehat{F}$ converge.

Demostración. Haciendo un cambio lineal de coordenadas, es suficiente mostrar que la convergencia de la serie formal

$$
\sum a_{i_{1}, \ldots, i_{n}} X_{1}^{i_{1}} \ldots X_{n}^{i_{n}}
$$

es equivalente a

$$
\sum a_{i_{1}, \ldots, i_{n}} X_{1}^{i_{1}+\cdots+i_{n}} X_{2}^{i_{2}} \ldots X_{n}^{i_{n}}
$$


La parte directa es inmediata, por composición, es decir,

$$
\begin{aligned}
\widehat{F}\left(X_{1}, X_{2}, \ldots, X_{n}\right) & =\sum a_{i_{1} i_{2} \ldots i_{n}} X_{1}^{i_{1}} X_{2}^{i_{2}} \ldots X_{n}^{i_{n}} \\
\widehat{F} \circ \pi\left(X_{1}, X_{2}, \ldots, X_{n}\right) & =\widehat{F}\left(X_{1}, X_{1} X_{2}, \ldots, X_{1} X_{n}\right) \\
& =\sum a_{i_{1} i_{2} \ldots i_{n}} X_{1}^{i_{1}}\left(X_{1} X_{2}\right)^{i_{2}} \ldots\left(X_{1} X_{n}\right)^{i_{n}} \\
& =\sum a_{i_{1} i_{2} \ldots i_{n}} X_{1}^{i_{1}+i_{2}+\ldots+i_{n}} X_{2}^{i_{2}} \ldots X_{n}^{i_{n}}
\end{aligned}
$$

Recíprocamente, la convergencia de la segunda serie da una constante $A>1$ tal que

$$
\left|a_{i_{1}, \ldots, i_{n}}\right| \leqslant A^{i_{1}+2\left(i_{2}+\cdots+i_{n}\right)}
$$

de lo cual $\left|a_{i_{1}, \ldots, i_{n}}\right| \leqslant\left(A^{2}\right)^{\left(i_{1}+\cdots+i_{n}\right)}$

$$
\begin{aligned}
\left|a_{i_{1}, \ldots, i_{n}}\right|\left|X_{1}\right|^{i_{1}}\left|X_{2}\right|^{i_{2}} \ldots\left|X_{n}\right|^{i_{n}} & \leqslant \frac{\left(A^{2}\right)^{\left(i_{1}+\cdots+i_{n}\right)}}{A^{n_{0} i_{1}} A^{n_{0} i_{2}} \ldots A^{n_{0} i_{n}}} \\
& =\frac{\left(A^{2}\right)^{\left(i_{1}+\cdots+i_{n}\right)}}{A^{n_{0}\left(i_{1}+\cdots+i_{n}\right)}} \\
& =\frac{\left(A^{2}\right)^{\left(i_{1}+\cdots+i_{n}\right)}}{\left(A^{2}\right)^{\frac{n_{0}}{2}\left(i_{1}+\cdots+i_{n}\right)}} \\
& =\frac{1}{\left(A^{2}\right)^{\left(\frac{n_{0}}{2}-1\right)\left(i_{1}+\cdots+i_{n}\right)}}
\end{aligned}
$$

y por el criterio $M$-test de Weierstrass la primera serie converge.

Proposición 6.2 Sea $\mathcal{F}$ un germen de foliación en el origen de $\mathbb{C}^{2}$ posee una integral primera formal $\widehat{f}=\widehat{f}_{1} \widehat{f}_{2}$ donde $\widehat{f}_{1}$ es irreducible y $\widehat{f}_{1}$ y $\widehat{f}_{2}$ no tienen factores comunes. Si existe $\widehat{\tau} \in t \mathbb{C}[[t]], \quad\left(\frac{\partial \widehat{\tau}}{\partial t}(0)\right) \neq 0$ tal que $\widehat{\tau} \circ \widehat{f}$ converge. En particular, $\mathcal{F}$ posee integral primera convergente.

Demostración. Haremos inducción sobre el número mínimo $q$ de explosiones necesarias para desingularizarla. Cuando $q=0$, aplicamos el lema anterior y la observación anterior. Supongamos que el resultado es cierto para $q-1$ explosiones necesarias para desingularizar $\mathcal{F}$. Sea $\pi: N^{\prime} \rightarrow \mathbb{C}^{2}$ explosión del origen de $\mathbb{C}^{2}$ y $P \in \pi^{-1}(0)$ el único punto del divisor excepcional por el que pasa el transformado estricto $\widehat{f}_{1}^{\prime}=0$ de la curva formal $\left(\widehat{f}_{1}=0\right)$. La inducción aplicada al germen en $P$ del transformado estricto $\pi^{*} \mathcal{F}$ de $\mathcal{F}$ y para $(\widehat{f} \circ \pi)_{P}=\widehat{f}_{1}^{\prime} \widehat{g}$; si existe $\widehat{\tau} \in \mathbb{C}[[t]]$ tal que $\widehat{\tau} \circ(\widehat{f} \circ \pi)_{P}$ convergente. La convergencia de $\widehat{\tau} \circ \widehat{f}$ se cumple por el lema anterior

La transversalidad permite extender a dimensiones mayores los resultados relativos a la existencia de integral primera convergente desde que hay una formal. El Algoritmo de GodbillonVey produce una integral formal, completando la demostración del Teorema de Malgrange.

Test de convergencia El siguiente lema permite reconocer la convergencia de integrales primeras de curvas paramétricas [5].

Lema 6.4 (Mattei- Moussu) Sea $\mathcal{F}$ un germen de foliación holomorfa en el origen de $\mathbb{C}^{n}$, posee integral primera formal $\hat{f}$. Suponiendo que existe $\gamma:(\mathbb{C}, 0) \rightarrow\left(\mathbb{C}^{n}, 0\right)$ tal que $\hat{f} \circ \gamma$ sea no constante y converja. Entonces $\widehat{f}$ converge.

Demostración. Sea $\pi: N^{\prime} \rightarrow \mathbb{C}^{n}$ las explosiones del origen y consideremos el levantamiento $\tilde{\gamma}:(\mathbb{C}, 0) \rightarrow N^{\prime}$ de $\gamma$ por la explosión $\pi$. Supongamos que $\tilde{\gamma}(0)=P \notin \operatorname{Sing}\left(\pi^{*} \mathcal{F}\right)$. El teorema de 
Frobenius clásico asegura la existencia de un sistema de coordenadas $y_{1}, \ldots, y_{n}$ en $P$ tal que $\pi^{*} \mathcal{F}$ es dado por $d y_{1}$; Por consiguiente $(\widehat{f} \circ \pi)_{P}=\hat{\ell}\left(y_{1}\right)$ donde $\hat{\ell} \in \mathbb{C}[[t]]$. Como $\hat{f} \circ \pi \circ \tilde{\gamma}=\hat{\ell}\left(y_{1}(\tilde{\gamma})\right)$ es convergente y no constante, $\hat{\ell}$ es convergente; así $(\widehat{f} \circ \pi)_{P}$ también lo es. El lema 6.3 implica la convergencia de $\hat{f}$. Si $P \in \operatorname{Sing}\left(\pi^{*} \mathcal{F}\right)$ se reduce al caso anterior por un número finito de explosiones siguiendo la dirección de la curva $\gamma$; si ambos cayeran en el conjunto de puntos singulares entonces $\gamma$ es una curva integral y $\widehat{f} \circ \gamma$ sería constante.

Definición 6.1 Sea $I \subset \mathcal{O}_{n}$ un ideal y consideremos el germen del conjunto analítico $X=\{f=0: f \in I\}$. La dimensión $\operatorname{dim} X$ es $\operatorname{dim} X=n-\operatorname{prof}(I)$.

Una foliación holomorfa $\mathcal{F}$ de codimensión 1 en $\left(\mathbb{C}^{n}, 0\right)$ es definida por una 1-forma holomorfa $\omega$ definida en $\left(\mathbb{C}^{n}, 0\right)$ por la distribución

$$
\operatorname{Ker}(\omega(p)) \quad \operatorname{con} \quad \omega(p) \neq 0
$$

El conjunto $\operatorname{Sing}(\mathcal{F})=\left\{p \in\left(\mathbb{C}^{n}, 0\right): \omega(p)=0\right\}$ es llamado el conjunto singular de $\mathcal{F}$.

Teorema 6.3 Sea $\mathcal{F}$ un germen de foliación holomorfa de codimensión 1 en el origen de $\left(\mathbb{C}^{n}, 0\right)$. Si Codim $(\operatorname{Sing} \mathcal{F}) \geqslant 3$, entonces $\mathcal{F}$ posee una integral primera convergente.

Demostración. Según el teorema de Frobenius formal, existe $\widehat{g}$ y $\widehat{f} \in \mathbb{C}\left[\left[x_{1}, \ldots, x_{n}\right]\right]$ tales que:

$$
\omega=\widehat{g} d \widehat{f}, \widehat{g}(0) \neq 0 .
$$

Si $i:\left(\mathbb{C}^{2}, 0\right) \rightarrow\left(\mathbb{C}^{n}, 0\right)$ es una inmersión transversal a $\mathcal{F}$ entonces:

$$
i^{*} \omega=(\widehat{g} \circ i) d(\widehat{f} \circ i)
$$

y $\widehat{f_{0}}=\widehat{f} \circ i$ satisface las hipótesis de la proposición 6.2 ; en efecto si $\widehat{f_{0}}$ no es reducida, la codimensión de $\operatorname{Sing}\left(d \widehat{f}_{0}\right)$ es 1 . Por consiguiente existe $\hat{\ell} \in t \mathbb{C}[[t]]$ tal que $\hat{\ell} \circ \widehat{f} \circ i$ converja. La convergencia de $\hat{\ell} \circ \widehat{f}$ resulta del lema 6.4. Aplicando en $\gamma=i \circ \gamma_{1}$ elegido donde

$$
\gamma_{1}:(\mathbb{C}, 0) \rightarrow\left(\mathbb{C}^{2}, 0\right)
$$

es tal que $\widehat{f} \circ \gamma$ sea no constante.

\section{Teorema de Malgrange sobre un subcuerpo $\mathbb{K}$ de los comple- jos}

Teorema 7.1 Sea $\omega$ un germen en $0 \in \mathbb{C}^{n}$ una 1-forma diferencial holomorfa de codimensión 1 con coeficientes en $\mathbb{K}\{x\}$, que verifica:

i) $\omega \wedge d \omega=0$

ii) $\operatorname{Codim}(\operatorname{Sing} \omega) \geq 3$.

Entonces $\omega$ es $\mathbb{K}$-integrable si existen dos gérmenes $f, g \in \mathbb{K}\{x\}$ tales que $\omega=g d f$ con $g(0) \neq 0$.

Demostración. Del Teorema de De Rham-Saito ([2], p.45) y la definición 6.1 la condición Codim $(\operatorname{Sing} \omega) \geq 3$ implica que la 1 -forma $\omega$ posee la propiedad de la división en $\Omega_{2}(\mathbb{C})$ : si $\alpha \in \Omega_{2}(\mathbb{C})$ verifica $\alpha \wedge \omega=0$, si existe $\beta \in \Omega_{1}(\mathbb{C})$ tal que $\alpha=\omega \wedge \beta$. Más precisamente, si 
$\alpha \in \Omega_{2}(\mathbb{K})$, podemos escoger $\beta \in \Omega_{1}(\mathbb{K})$. En efecto los coeficientes de $\beta$ en la base $d x_{1}, d x_{2}, \ldots, d x_{n}$ son soluciones lineales con coeficientes en $\mathbb{K}\{x\}$, es decir,

$$
\begin{aligned}
\omega & =\sum_{I} a_{I} d x_{I} \\
\beta & =\sum_{I} b_{I} d x_{I} \\
\alpha & =\sum_{I} \sum_{J} c_{I J} d x_{I} \wedge d x_{J} \\
c_{i i} & =a_{i} b_{i}=0 \\
c_{i j} & =\left(a_{i} b_{j}-b_{i} a_{j}\right), \quad i<j, \quad i, j=1, \ldots, n
\end{aligned}
$$

En particular, debemos dividir por ciertas determinantes $\Delta(x) \in \mathbb{K}\{x\}$ con $\Delta(0) \neq 0$. La inversa de tal elemento en $\mathbb{K}\{x\}$ es también un elemento de $\mathbb{K}\{x\}$.

La 1-forma $\omega$ posee un algoritmo de Godbillon-Vey $\left\{\omega_{0}=\omega, \omega_{1}, \omega_{2}, \ldots\right\}$ donde los $\omega_{i} \in \mathbb{K}\{x\}$, es decir, $\omega \wedge d \omega=0$, existe $\omega_{1}$ tal que $d \omega=\omega \wedge \omega_{1}$, aplicando el diferencial exterior

$d^{2} \omega=d \omega \wedge \omega_{1}-\omega \wedge d \omega_{1}$ reemplazando $d \omega$ en la relación anterior obtenemos $0=\left(\omega \wedge \omega_{1}\right) \wedge \omega_{1}-\omega \wedge d \omega_{1}$

$$
\omega \wedge d \omega_{1}=0
$$

Existe $\omega_{2}$ tales que $d \omega_{1}=\omega \wedge \omega_{2}$, aplicando el diferencial exterior, $0=d \omega \wedge \omega_{2}-\omega \wedge d \omega_{2}$ reemplazando $d \omega$ obtenemos $0=\omega \wedge \omega_{1} \wedge \omega_{2}-\omega \wedge d \omega_{2}=\omega \wedge\left(\omega_{1} \wedge \omega_{2}-d \omega_{2}\right)$. Así, existe $\omega_{3}$ tal que $\omega_{1} \wedge \omega_{2}-d \omega_{2}=\omega \wedge \omega_{3}$ agrupando $d \omega_{2}=\omega_{1} \wedge \omega_{2}+\omega \wedge \omega_{3}$ siguiendo inductivamente con $n \geq 0, \omega=\omega_{0}$ obtenemos

$$
d \omega_{n}=\omega_{0} \wedge \omega_{n+1}+\sum_{1 \leq k \leq n}\left(\begin{array}{l}
n \\
k
\end{array}\right) \omega_{k} \wedge \omega_{n-k+1} .
$$

Ahora podemos añadir una variable $t$ y considerar la 1-forma diferencial formal

$$
\begin{aligned}
\Pi & =d t+\omega+t \omega_{1}+\frac{t^{2}}{2 !} \omega_{2}+\cdots+\frac{t^{n}}{n !} \omega_{n}+\cdots \\
& =d t+\sum_{n=0}^{\infty} \frac{t^{n}}{n !} \omega_{n} \\
d \Pi & =\sum_{n=0}^{\infty} \frac{t^{n}}{n !}\left(\omega \wedge \omega_{n+1}+\sum_{k=1}^{n}\left(\begin{array}{l}
n \\
k
\end{array}\right) \omega_{k} \wedge \omega_{n-k+1}\right)+d t \wedge \sum_{n=1}^{\infty} \frac{t^{n-1}}{(n-1) !} \omega_{n} \\
& =\sum_{n=0}^{\infty} \frac{t^{n}}{n !} \omega \wedge \omega_{n+1}+\sum_{n=0}^{\infty} \frac{t^{n}}{n !} \sum_{k=1}^{n}\left(\begin{array}{l}
n \\
k
\end{array}\right) \omega_{k} \wedge \omega_{n-k+1}+d t \wedge \sum_{n=1}^{\infty} \frac{t^{n-1}}{(n-1) !} \omega_{n} \\
& =\sum_{n=1}^{\infty} \frac{t^{n-1}}{(n-1) !} \omega \wedge \omega_{n}+d t \wedge \sum_{n=1}^{\infty} \frac{t^{n-1}}{(n-1) !} \omega_{n}+\left(\sum_{n=1}^{\infty} \frac{t^{n}}{n !} \omega_{n}\right) \wedge\left(\sum_{n=1}^{\infty} \frac{t^{n-1}}{(n-1) !} \omega_{n}\right) \\
& =\sum_{n=1}^{\infty} \frac{t^{n-1}}{(n-1) !} \omega \wedge \omega_{n}+d t \wedge \sum_{n=1}^{\infty} \frac{t^{n-1}}{(n-1) !} \omega_{n}+\sum_{n=1}^{\infty}\left(\sum_{k=1}^{n-1}\left(\frac{t^{k}}{k !} \omega_{k}\right) \wedge\left(\frac{t^{n-k-1}}{(n-k-1) !} \omega_{n-k}\right)\right) .
\end{aligned}
$$

Se verifica que $\Pi \wedge d \Pi=0$ y $\Pi(0) \neq 0$. Según el teorema de Frobenius Formal sobre $\mathbb{K}$, existen $\hat{F}, \hat{G} \in \mathbb{K}[[x, t]]$ tales que $\Pi=\hat{G} d \hat{F}, \hat{G}(\mathbf{0}, 0)=0$. Haciendo $t=0$ en esta relación obtenemos:

$$
\omega=\hat{g} d \hat{f} \operatorname{con} \hat{g}, \hat{f} \in \mathbb{K}[[x]], \quad \hat{g}(0) \neq 0 .
$$


Suponemos que $\hat{f}=f^{\nu}+f^{\nu+1}+\cdots$ con $f^{\nu} \neq 0$. Si existe un vector $v \in \mathbb{C}^{n}$ entonces

$$
d f^{\nu}(v)=\nu f^{\nu}(v) \neq 0
$$

En efecto,

$$
\begin{aligned}
d_{x} f^{\nu} & =\frac{\partial f^{\nu}}{\partial x_{1}} d x_{1}+\frac{\partial f^{\nu}}{\partial x_{2}} d x_{2}+\cdots+\frac{\partial f^{\nu}}{\partial x_{n}} d x_{n} \\
d_{x} f^{\nu}(v) & =\frac{\partial f^{\nu}(x)}{\partial x_{1}} d x_{1}(v)+\frac{\partial f^{\nu}(x)}{\partial x_{2}} d x_{2}(v)+\cdots+\frac{\partial f^{\nu}(v)}{\partial x_{n}} d x_{n}(v) \\
& =\frac{\partial f^{\nu}(x)}{\partial x_{1}} v_{1}+\frac{\partial f^{\nu}(x)}{\partial x_{2}} v_{2}+\cdots+\frac{\partial f^{\nu}(x)}{\partial x_{n}} v_{n}, \quad v=x \\
& =\frac{\partial f^{\nu}(x)}{\partial x_{1}} x_{1}+\frac{\partial f^{\nu}(x)}{\partial x_{2}} x_{2}+\cdots+\frac{\partial f^{\nu}(x)}{\partial x_{n}} x_{n} \\
& =\nu f^{\nu}(x) \neq 0
\end{aligned}
$$

Mediante un cambio de coordenadas $\mathbb{K}$-lineal podemos suponer que $v=(1,0, \ldots, 0)$ y también que:

$$
\begin{aligned}
\hat{f}(x, & 0, \ldots, 0)=\hat{f}_{1}\left(x_{1}\right)=\lambda x_{1}^{\nu}\left(1+\varphi_{1}\left(x_{1}\right)\right), \varphi_{1} \in \mathbb{K}\left[\left[x_{1}\right]\right], \varphi_{1}(0)=0, \lambda \in \mathbb{K} . \\
\hat{f}_{1}\left(x_{1}\right) & =\hat{f}\left(x_{1}, 0, \ldots, 0\right) \\
& =f^{\nu}\left(x_{1}, 0, \ldots, 0\right)+f^{\nu+1}\left(x_{1}, 0, \ldots, 0\right)+\cdots \\
& =x_{1}^{\nu} f^{\nu}(1,0, \ldots, 0)+x_{1}^{\nu+1} f^{\nu+1}(1,0, \ldots, 0)+x_{1}^{\nu+2} f^{\nu+2}(1,0, \ldots, 0)+\cdots \\
& =x_{1}^{\nu} \lambda+x_{1}^{\nu}\left(x_{1} f^{\nu+1}(1,0, \ldots, 0)+x_{1}^{2} f^{\nu+2}(1,0, \ldots, 0)+\cdots\right) \\
& =\lambda x_{1}^{\nu}\left(1+\varphi_{1}\left(x_{1}\right)\right)
\end{aligned}
$$

Multiplicamos $\hat{f}$ por $\frac{1}{\lambda}$ y $\hat{g}$ por $\lambda\left(\omega=\hat{g} d \hat{f}=(\lambda \hat{g})\left(d\left(\frac{1}{\lambda} \cdot \hat{f}\right)\right)\right)$ podemos suponer $\lambda=1$. El elemento $1+\varphi_{1}$ posee una raíz $\nu$-ésima en $\mathbb{K}\left[\left[x_{1}\right]\right]$ del tipo $1+\varphi_{2}$. Para terminar la prueba del teorema, debemos verificar la siguiente afirmación:

$\left.A_{1}\right)$ Si existe $\ell \in \mathbb{K}\left[\left[x_{1}\right]\right], \ell^{\prime}(0) \neq 0$, tal que $\ell_{\circ} \hat{f}_{1}\left(x_{1}\right) \in \mathbb{K}\left\{x_{1}\right\}$.

En efecto, si existe $\rho>0$ tal que la foliación $\mathcal{F}$ definida por $\omega=0$ en $0<\|x\|<\rho$ es transversal a la curva $x_{1} \mapsto\left(x_{1}, 0, \ldots, 0\right)$, y $\ell \circ \hat{f}$ es una integral primera de $\omega$ que posee las propiedades requeridas. Para la prueba de la afirmación $A_{1}$ ) usaremos argumentos de [5]. Sea $H_{\mathbb{K}}\left(\hat{f}_{1}\right)$ el grupo de invarianza de $\hat{f}_{1}$ en $\mathbb{K}\left[\left[x_{1}\right]\right]$ :

$$
H_{\mathbb{K}}\left(\hat{f}_{1}\right)=\left\{\hat{h} \in \mathbb{K}\left[\left[x_{1}\right]\right]: \hat{f}_{1} \circ \hat{h}=\hat{f}_{1}, \hat{h}^{\prime}(0) \neq 0\right\} .
$$

mostraremos como en [5] la siguiente afirmación:

$\left.A_{2}\right)$ Si $H_{\mathbb{K}}\left(\hat{f}_{1}\right)$ esta contenido en $\mathbb{K}\left\{x_{1}\right\}$, la afirmación $\left.A_{1}\right)$ es verdadera.

$\mathrm{O} A_{2}$ ) es verdadera desde que $\hat{f}$ no es una potencia, y es el caso. En efecto, puesto que $\omega=\hat{g} d \hat{f}$ con $\hat{g}(0) \neq 0$, el ideal generado por los coeficientes de las derivadas parciales de $\hat{f}$ en $\mathbb{K}[[x]]$ es el mismo que el ideal engendrado por los coeficientes de $\omega$ en la base $d x_{1}, d x_{2}, \ldots, d x_{n}$. Con la hipótesis $\operatorname{Codim}(\operatorname{Sing}(\omega)) \geq 3$ tendría altura 3 , no sería el caso si $\hat{f}$ fuera una potencia. 


\section{Agradecimientos}

- El primer autor agradece el financiamiento de la Pontificia Universidad Católica del Perú mediante el proyecto VRI-DGI 2018-1-0059.

- El segundo autor agradece el financiamiento de la Universidad Nacional Agraria La Molina.

\section{Conclusión}

El aporte del presente trabajo es de carácter teórico, se espera que este artículo sirva como referencia para futuros trabajos de investigación.

\section{Referencias bibliográficas}

[1] Apaza, D. (2012). El teorema de De Rham - Saito y el Teorema de Frobenius Singular (Tesis de Maestría). Pontificia Universidad Católica del Perú, Lima, Perú.

[2] Apaza, D. (2012). El teorema de De Rham - Saito. Pro mathematica, 26(51-52), 49-74.

[3] Malgrange, B.(1976). Frobenius avec Singularités 1. Codimension un. Publications Mathématiques de l'Institut des Hautes Études Scientifiques, 46(1), 163-173.

[4] Matsushima, Y.(1972). Differentiable Manifolds.New York, State United: Marcel Dekker,Inc.

[5] Mattei, J. F.; Moussu, R. (1980). Holonomie et intégrales premières. Annales scientifiques de l'École Normale Supérieure, Série 4, Tome 13 (1980) no. 4, pp. 469-523.

[6] Moussu, R. y Rolin, J. P.(2009). Une Prueve Combinatoire du Théorème de Frobenius. In Équations différentielles et singularités. En l'honneur de J. M. Aroca, Astérisque, no. 323, pp. 253-260.

[7] Moussu, R. y Tougeron, J. C. (1976). Fonctions composées analytiques et différentiables. C. R. Acad. Sc. Paris, t.282, 1237-1240.

[8] Saito, K.(1973). Calcul algébrique de la monodromie. In Singularités à Cargèse, Astérisque, no. 7-8, pp. 195-211.

[9] Saito, K. (1976). On Generalisation of de Rham Lemma. Annales de l'Institut Fourier, $26(2), 165-170$. 